\title{
An amphipathic sequence in the cytoplasmic tail of HIV-1 Env alters cell tropism and modulates viral receptor specificity
}

\author{
A. N. VZOROV, C. YANG, R. W. COMPANS
}

Department of Microbiology and Immunology and Emory Vaccine Center Emory University School of Medicine, 1518 Clifton Rd, Room 5005, Atlanta, GA 30322, USA

Received February 6, 2015; revised April 16, 2015; accepted July 27, 2015

\begin{abstract}
Summary. - The human immunodeficiency virus type 1 (HIV-1) 92UG046 Env protein, obtained from a CD4independent HIV-1 primary isolate (Zerhouni et al., 2004), has the ability to initiate an infection in HeLa cells expressing CD4 when carrying the full-length (FL) Env, but uses CD8 molecules for receptor-mediated entry when carrying a truncated Env (CT84). To determine whether a specific length or structure in the cytoplasmic tail (CT) is responsible for this alteration of tropism, we compared a series of Env constructs with different CT truncations and the presence or absence of an amphipathic alpha- helical sequence. We found that truncated constructs containing the alpha-helical LLP-2 structure in their CT domains conferred a switch from CD4 to CD8 tropism. The results support the conclusion that the structure of the $\mathrm{CT}$ domain can play an important role in determining receptor specificity.
\end{abstract}

Keywords: HIV-1; Env glycoprotein; gp41; gp120; cytoplasmic tail truncation; alpha-helical LLP2 sequence; CD4, CD8; receptor specificity

\section{Introduction}

Prevention and treatment of infection induced by human immunodeficiency virus type $1(\mathrm{HIV}-1)$ is a worldwide public health goal, and a better understanding of HIV-1 tropism is important for development of HIV-1 inhibitors and vaccines. HIV and simian immunodeficiency virus (SIV) Env glycoproteins possess an unusually long cytoplasmic tail (CT) sequence, and acquisition of CT truncations by premature stop codons, which remove almost the entire CT, is a potential mechanism used by HIV or SIV for adaptation to new cell types or escape from effects of entry inhibitors (Kodama et al., 1989; Mulligan et al., 1992; Tilton and Doms, 2010; Waheed et al., 2007). The size and sequence of the gp41 cytoplasmic domain can also modify the biological properties

E-mail: avzorov@emory.edu; phone: +1-404-727-3228.

Abbreviations: $\mathrm{CT}=$ cytoplasmic tail; $\mathrm{FL}=$ full-length; $\mathrm{HIV}-1=$ human immunodeficiency virus type 1 ; LLP-1,-2,-3 = conserved alpha-helical "lentivirus lytic peptide" domains; $\mathrm{SP}=$ signal peptide sequence; $\mathrm{SIV}=$ simian immunodeficiency virus; $\mathrm{SU}(\mathrm{s})=$ surface subunit(s); $\mathrm{TM}=$ transmembrane protein; $\mathrm{WT}=$ wild type of the Env protein (Vzorov and Compans, 2011). Although SIVmac239 virus with a FL Env is lymphocytotropic, infection of macaques by a molecular clone SIV/17E-Fr, with a truncated cytoplasmic tail of 48 aa, led to disseminated infection (Mankowski et al., 1997). The cytoplasmic tail of the transmembrane protein (TM) also has an essential role in other important viral functions (Berlioz-Torrent et al., 1999; Bowers et al., 2000; Sauter et al., 1996) and contains multiple motifs including three highly conserved alpha-helical "lentivirus lytic peptide" domains (LLP-1, LLP-2, LLP-3) engaged in association with the plasma membrane, reducing bilayer stability, changing membrane ion permeability, and mediating cytopathicity (Chen et al., 2001; Chernomordik et al., 1994; Comardelle et al., 1997; Kalia et al., 2003; Miller et al., 1993; Srinivas et al., 1992; Venable et al., 1989).

CD4-independent variants of HIV-1 were reported with FL and truncated Envs coexisting as quasispecies, which were able to infect $\mathrm{CD}^{+}$or $\mathrm{CD}^{+}$cells (Zerhouni et al., 2004). A truncated cytoplasmic tail (84 aa) due to a point mutation in TM was observed in an HIV-1 T8-tropic isolate, and patients with elevated levels of such truncated Env variants showed more rapid progression of infection, and greater resistance to antiviral therapy (Waheed et al., 2007; Zerhouni et al., 2004). 
In the present study, we used cloned HIV-1 92UG046 Envs and viral pseudotypes to investigate the structural features of HIV-1 92UG046 Env-CT84, with a truncated 84 amino acid cytoplasmic tail, and the biological properties of HIV-1 with this naturally occurring truncated Env, and compared them with HIV-1 92UG046, which possesses the FL CT. We further investigated different forms of HIV-1 92 UG046 Env, including mutants with different lengths and structure of CT domains, on their incorporation into pseudotyped virions, syncytia formation activity and receptor/coreceptor mediated entry.

\section{Materials and Methods}

Cells. 3T3T4X4, 3T3T4R5, NIH3T3, HeLaT4, HeLaT8 and HeLa cells were obtained from the AIDS Research and Reference Reagent Program (ARRRP), Division of AIDS (NIH). TZM-bl cells (derived by J. Kappes and X.Wu and contributed by Tranzyme, Inc. Durham, NC) (Wei et al., 2002) were obtained through the NIH ARRRP. Hep2, CV-1, 3T3T4X4, 3T3T4R5, NIH3T3, 293T, HeLaT4, HeLaT8, HeLa, and TZM-bl cells were maintained as described (Vzorov et al., 2007). Human blood samples were obtained from volunteers enrolled in the Emory University Institutional Review Board approved "Emory Vaccine Center's Healthy Adults Study" (Protocol \#555-2000). Volunteers in this study were healthy adults, aged from 23 to 62, who had signed a written informed consent form. Human PBMCs were separated by centrifugation of whole blood using LSM Lymphocyte Separation Medium (ICN Biomedicals Inc., Costa Mesa, CA). Cells were then stimulated with concanavalin A (Con A, $5 \mu \mathrm{g} / \mathrm{ml}$ in RPMI 1640 containing 10\% heat-inactivated fetal calf serum; human interleukin 2 (hIL-2), $10 \mathrm{U} / \mathrm{ml} ; 10 \mathrm{mmol} / \mathrm{l} \mathrm{HEPES}$; and antibiotics) for 2 days before infection.

Plasmids and viruses. For molecular cloning, cell-free HIV-1 92UG046 (GenBank Acc. No. AY623600) was obtained from the NIH ARRRP. HIV-1 DNA was extracted from infected human PBMCs using a DNeasy Tissue kit (Qiagen, Valencia, CA) and the env gene sequence was amplified by PCR. Two types of 92UG046 Env constructs were compared: wild type (WT) constructs with original rev and signal peptide (SP) sequences; and modified constructs with the rev and SP replaced by those of NL4-3, which were used to optimize the expression of FL and truncated Envs. The sequence of the env gene was identical to that of a CD4-independent HIV-1 clone (92UG046-T8) with a truncated Env protein (GeneBank: AY623600), with several mutations occurring after cell culture passage: K4M, N17D (SP sequence); A461T (gp120); and M620I, K774R (gp41). The FL Env has a mutation in a stop codon at position 784 to a tryptophan (trp) codon. Recombinant vaccinia virus vTF7-3 and the wild type vaccinia virus strain IHD-J were kindly provided by Bernard Moss (NIH, Bethesda, MD) and were propagated and titrated on CV-1 cells. The plasmid pGINT7 $\beta$-Gal was provided by Edward Berger (NIH). The plasmid pSG3 delta
Env backbone and the pNL4-3.Luc.R.E- delta Env, Vpr backbone with the luciferase gene inserted into the pNL4-3 nef gene were obtained from the NIH ARRRP. The plasmid vector pCAGGS (kindly provided by Y. Kawaoka Madison, WI) containing a CMV/ $\beta$-actin chimeric promoter or pcDNA3.1(+) (Invitrogen Corporation, Camarillo, CA), were used to express Env constructs. The genes encoding the Rev and FL Env protein of 92UG046 virus were amplified and inserted in the plasmid vector for transient transfection experiments.

Conventional and real-time PCR amplification for HIV. Conventional and real-time PCR amplification were used to determine the entry of pseudotyped virions with FL or truncated Envs. Quantification of proviral DNA from infected cells was performed by real-time PCR using the TaqMan amplification system as described (Kim et al., 2001; Schmidtmayerova et al., 1998; Vzorov et al., 2007). For PCR amplification of the HIV-1 gag and 2-LTR $\mathrm{U} 5 / \mathrm{U} 3$ regions, primers and probes modified with the fluorescent dye FAM were obtained from Life Technologies (Grand-Island, NY). For the HIV-1 gag region, forward and reverse PCR primers were HIVgagF 5'-AGGACTCGGCTTGCTGAAG-3' and HIVgagR 5'-GCCTCCGCTAGTCAAAATTTTTGG-3', respectively. The HIVgag probe 5'-CCCCTCGCCTCTTGC-3' was modified with the fluorescent dye FAM. For the HIV 2LTR U5/U3 regions, PCR was carried out using forward and reverse PCR primers 2LTRSG3U5/U3F 5'-TCCCTCATACCCTTTTAGTCAGTGT-3' and 2LTRSG3U5/U3R 5'-CAAGGATATCTTGTCTTCTTT GGGAGAA-3'. The fluorogenic 2LTRSG3U5/U3 probe used was 5'-TTCCATGCTAGAGATTTT-3'. DNA samples corresponding to equal numbers of cells infected by pseudotyped virions were analyzed in parallel. Fluorescence was recorded as a function of PCR amplification cycle. Quantitative HIV determinations were made by comparison with a standard curve produced by using serial dilutions of plasmid DNA (Desire et al., 2001).

Quantitative real-time PCR analysis. The reaction was carried out on a 96-well optical plate (Life Technologies) in a $20 \mu$ reaction volume containing $1 / 20$ of the total DNA extracted from $3 \times 10^{5}$ cells using TaqMan Universal PCR Mastermix (Roche, Branchburg, NJ). All sequences were amplified using the Applied Biosystems 7500 Real-Time PCR System with the program: $50^{\circ} \mathrm{C}$ for $2 \mathrm{~min}, 95^{\circ} \mathrm{C}$ for $10 \mathrm{~min}$, followed by 45 cycles at $95^{\circ} \mathrm{C}$ for $15 \mathrm{sec}$, and $60^{\circ} \mathrm{C}$ for $1 \mathrm{~min}$. Samples were tested in duplicate in parallel with the housekeeping gene alpha-tubulin, using primers and probes modified with the fluorescent dye FAM (obtained from Life Technologies). For relative quantitation, delta-delta Ct analysis (Desire et al., 2001) was applied to calculate the fold differences between samples.

Analysis of protein expression. Protein expression was carried out using the recombinant vaccinia virus T7 transient expression system. Briefly, Hep2 cells were seeded in culture dishes and grown to $90 \%$ confluence overnight. The cells were then infected with recombinant vaccinia virus VTF7-3 (which expresses the T7 polymerase) at a multiplicity of infection (MOI) of 0.1 for $2 \mathrm{hr}$ followed by transfection with indicated DNA constructs using FuGene 6, obtained from Roche (Indianapolis, IN) At $40 \mathrm{hr}$ post-infection/ 
transfection, cells were starved in Met, Cys-deficient DMEM for $30 \mathrm{~min}$, and then labeled with $\left[{ }^{35} \mathrm{~S}\right]$-Met, Cys-labeling mix (obtained from Amersham, Piscataway, NJ) for 3 hr. Surface expression of the HIV Env protein was detected by a surface biotinylation assay as described previously (Vzorov and Compans, 2011). Env expression levels were determined with polyclonal antibodies (HIV-Ig) and Western blotting (WB) or Radioimmunoprecipitation (RIP) methods.

Colorimetric lysate cell fusion assay. Dishes (60-mm diameter) of subconfluent NIH3T3 cells were infected for $1 \mathrm{hr}$ with the vTF7-3 virus at an MOI of 1-2 and then transfected with $4 \mu \mathrm{g}$ of plasmids for expression of Env constructs. A second population of 3T3T4R5 cells was infected with wt vaccinia virus strain IHD-J and transfected with the pGINT7 $B-G a l$ plasmid, which contains the $ß$-galactosidase ( $B$-Gal) gene under the control of the T7 promoter. At 16 to $20 \mathrm{hr}$ post-transfection, the two cell populations were suspended, mixed in a 96-well tissue culture plate and incubated for $2 \mathrm{hr} 30 \mathrm{~min}$ at $37^{\circ} \mathrm{C}$, after which cell fusion was quantitated by a colorimetric lysate assay (Nussbaum et al., 1994). The data were analyzed with the Delta Soft II Microplate analysis program.

Pseudotype virus generation. Pseudotyped virions were produced by co-transfection of 293T cells with a pSG3 delta Env or pNL4-3. Luc.R.E- delta Env, Vpr backbone and Env constructs using calcium phosphate precipitation. After 3 days, supernatants were clarified by centrifugation at $3500 \mathrm{rpm}$ for $15 \mathrm{~min}$ and analyzed by titration in TZM-bl cells or RT-PCR in HeLaT4/HeLaT8 cells. Concentrated stocks of pseudotyped virions were prepared by filtration through a $0.45 \mu \mathrm{m}$ syringe filter and pelleting by centrifugation, and stored at $-80^{\circ} \mathrm{C}$ until use. Prior to infection, pseudotyped virions were treated with $200 \mathrm{U} / \mathrm{ml}$ RNase-free DNase I in growth medium containing $10 \mathrm{mmol} / \mathrm{l} \mathrm{MgCl}_{2}$ for $30 \mathrm{~min} 37^{\circ} \mathrm{C}$ to remove any contaminating proviral DNA.

Analysis of Env protein incorporation into pseudotyped viruses. Samples were analyzed by SDS-PAGE on $8 \%, 10 \%$ and $4-15 \%$ acrylamide gels and Western blotting using polyclonal human plasma antibody (HIV-Ig) for analysis of Gag and Env proteins and developed by an ECL kit obtained from Amersham (Piscataway, $\mathrm{NJ}$ ). The amounts of proteins were quantitated by densitometer analysis (NIH Image version 1.54).

Virus infectivity and neutralization assays. A single-cycle infectivity assay (Kimpton and Emerman, 1992) using Env pseudotyped virus and TZM-bl cells was used to assess the infectivity and neutralization capacity of the FL 92UG046 variant, as previously described (Vzorov and Compans, 2000). Infectivity was also measured $24 \mathrm{hr}$ post-infection by conventional PCR and monitored by quantitative real-time PCR. We used the infectious index (IU/ng), which is the ratio between DNA number copy and core antigen, to compare infectivity titers.

CD4/CD8 receptor blocking assay.At $24 \mathrm{hr}$ before infection, HeLaT4, HeLaT8 or TZM-bl cells $\left(7 \times 10^{4}\right.$ cells/60 mm dish treated and $6 \times 10^{4}$ cells $/ 60 \mathrm{~mm}$ dish untreated with $2 \mu \mathrm{g} / \mathrm{ml}$ aphidicolin) (Huberman, 1981) were pre-incubated with $1 \mu \mathrm{g} / \mathrm{ml}$ or $5 \mu \mathrm{g} / \mathrm{ml}$ of mouse anti-CD4 clone RPA-T4, isotype control (IgG1) (In- vitrogen Corporation, Camarillo, CA), mouse anti-CD4 clone Q4120 (Sigma-Aldrich, St. Louis, MO) or anti-CD8 clone B9.11 (Beckman Coulter, Brea, CA) for $1 \mathrm{hr}$ at $4^{\circ} \mathrm{C}$. Antibody-treated and untreated cells $\left(3 \times 10^{5}\right)$ were inoculated with CT84 or FL 92UG046 Env pseudotyped virions with an infectious index (IU/ng) of 1 or 10 , respectively, in medium with $2 \mu \mathrm{g} / \mathrm{ml}$ aphidicolin and $15 \mu \mathrm{g} / \mathrm{ml}$ DEAE-dextran. After $24 \mathrm{hr}$, DNA samples prepared from HeLaT4 or HeLaT8 cells were used for PCR or RT-PCR analysis. Infectious viral units in TZM-bl cells were determined by counting Tat-dependent $\beta$-gal expression-positive infected cells colonies by microscopy.

Competitive binding ELISA assay. ELISA plates were coated with $2 \mu \mathrm{g} / \mathrm{ml}$ of a murine monoclonal antibody against CD4 (OKT4) or CD8 (OKT8) (eBioscience, San Diego, CA) as described before (Vzorov and Compans, 2011). A 100- $\mu$ l volume (corresponding to $1 \mu \mathrm{g} / \mathrm{ml}$ ) of human recombinant CD4 (Protein Sciences Corp., Meriden, CT) or CD8a (22aa-131aa) (Abnova, Walnut, CA) was added per well and incubated for $2 \mathrm{hr}$. After five washes with PBS containing $0.05 \%$ Tween 20 , plates were incubated with a mixture of pseudotyped virions and anti-CD4 (clones RPA-T4, Q4120), or anti-CD8 (clone B9.11), or control IgG1 antibodies in assay diluent (ZeptoMetrix corporation, Buffalo, NY). After washes the captured pseudotyped virions were probed with biotin-conjugated polyclonal antibody to HIV-1. The 96-wells plates were developed with tetramethylbenzidine (TMB) and absorbance (OD) determined at $450 \mathrm{~nm}$.

Statistical analysis. Statistical analyses of means with standard deviation (SD) were performed using GraphPad Prism version 5.0 (GraphPad Software Inc.)

\section{Results}

Construction of HIV-1 Env mutants with different CT lengths

To examine whether the length of the cytoplasmic tail affects the biological properties of the Env protein, we compared 92UG046 env genes with a FL CT, the CT-84 truncation, and a short cytoplasmic tail CT-17 (Fig. 1). We also constructed modified env genes, in which the LLP-2 region was fused to the C-terminus of the truncated 92UG046 Env CT17 or to the C-terminus of 92UG046 Env CT62 (with a truncation located prior to LLP-2). In addition four conserved isoleucine/leucine residues of LLP2 were converted to alanine in the constructs designated CT17LLP2-4A or CT62LLP2-4A (Fig. 1).

\section{Effects of modification of CT domains on Env cell surface} expression

The synthesis and processing kinetics of the truncated Env proteins were similar to those of FL wild type Env. 


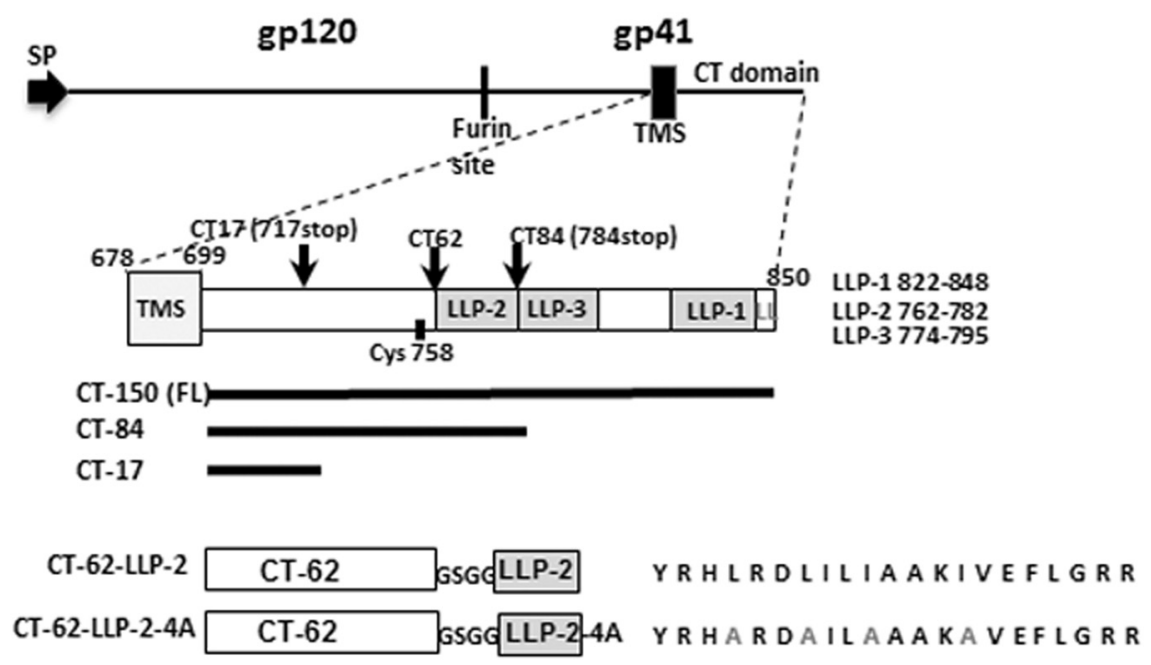

\author{
CT-17-LLP-2 CT-17 GSGGLLP-2 YRHLRDLILIA A K IVEFLGRR \\ CT-17-LLP-2-4A CT-17 GSG LLP-2-4A YR HAR DA IL A A A K A VEFLGRR
}

Fig. 1

Schematic diagram of the FL and truncated CT domains of 92UG046 Env

(Top) FL HIV-1 92UG046 envelope glycoprotein containing the signal peptide (SP), the furin cleavage site, the gp120 surface (SU) and gp41 transmembrane (TM) subunits. The CT domain is shown with five membrane-interactimg regions depicted. The upper sequence is LLP-2; the bottom sequence is the LLP2-4A mutant with A residues substituted for 4 conserved L and I residues. CT sequences of Env constructs are indicated as white boxes. Transmembrane-spanning domain (TMS) and LLP sequences are shown as gray boxes. A linker between CT domains (761 Ser/CT84; 716 Pro/CT17) and LLP sequences is GSGG. The positions of stop codons for CT84 and CT17 and the last C-terminal residue of CT62 are shown by arrows. The FL CT domain is a result of cell culture mutation of a stop codon at position 784 to a tryptophan (trp) codon (Zerhouni et al., 2004).

The ratio of SUs to TM subunits expressed on cell surfaces was also similar for all constructs including the wild type 92UG046 Env (not shown). The truncated Env protein CT17 was expressed on the cell surface at levels similar to that of the FL Env as determined by surface biotinylation and quantitation by phosphorimager analysis (Fig. 2a,b). The CT84 Env was expressed at $86 \%$ of the level of FL Env. The mutant glycoproteins with $4 \mathrm{~A}$ substitutions in their CT domains were expressed on the cell surface at similar levels as those observed with CT62LLP2 Env. The lowest surface expression level was observed with CT17LLP2 Env, which was about $33 \%$ of the CT62LLP2 level (Fig. 2c,d). The results show that most truncated Env proteins exhibit efficient transport and cell surface expression. However, the addition of the LLP2 domain to CT17 impaired its transport and cell surface expression.

\section{Syncytia formation activity of FL and truncated Envs}

To compare cell-cell fusion activity of the Env proteins and to determine the co-receptor usage, the vaccinia T7 expression system was used to express WT and mutant Env glycoproteins in NIH3T3 cells and determine expression of a $\beta$-Gal reporter gene in target cells (3T3T4X4 or 3T3T4R5) with HIV-specific receptors and coreceptors (CD4, CXCR4 or CD4, CCR5). No cell fusion was observed after expression of any of the Env proteins in 3T3T4R5 cells (Fig. 3). The CT17 truncated Env exhibited about $20 \%$ of the fusion activity of FL Env in 3T3T4X4 cells, whereas the CT84 Env did not exhibit any fusion activity using these target cells (Fig. 3). Taken together, the results show differences between the FL, CT84 and CT17 truncated Envs: CT84 Env exhibited a profound reduction of cell-cell fusion activity in $\mathrm{CD} 4^{+}$cells, whereas FL (WT Env) exhibited high and CT17 Env showed low syncytia formation activity. The results also indicate that 92UG046 Envs with FL or truncated CTs exhibit CXCR4 coreceptor usage.

Effects of CT domains on Env incorporation into pseudotyped virions

To evaluate the effects on Env incorporation into pseudotyped virions, we compared Env constructs with FL and truncated CT domains. We transfected 293T cells and three days post-transfection, supernatants from equal amounts of cells were analyzed. The replacement of WT with NL4-3 SP 
(a)

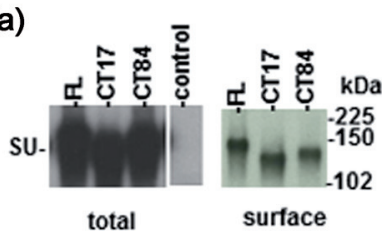

(c)

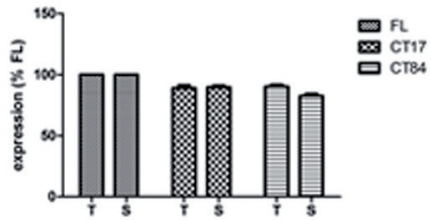

(b)

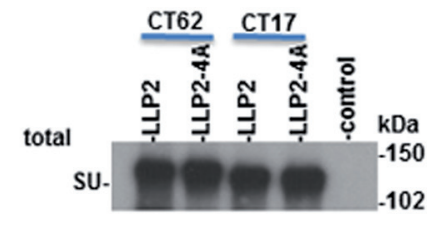

total

(short exposure)

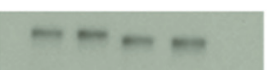

surface

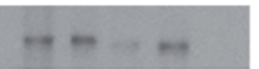

(d)

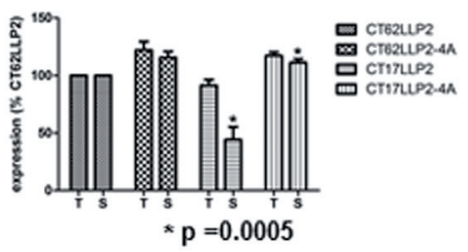

Fig. 2

Surface (S) and total (T) cell expression of FL and truncated Env proteins (92UG046) in Hep2 cells

Hep2 cells were infected by vaccinia vTF7-3 virus and then transfected by T7 plasmid constructs. At the end of the labeling period, the cell surface proteins were biotinylated, lysed and analyzed as described in Methods. All 92UG046 Env constructs (a) or modified 92UG046 Env constructs (c) possess the rev and env signal peptide of NL4-3 HIV-1. The levels of total or surface proteins (SU of Env) present in the autoradiograph relative to the FL 92 UG046 (b) or relative to CT62LLP2 (d) were quantitated by phosphorimager analysis as described in Methods. Data in c and d are plotted as the means of three experiments. Error bars represent standard deviations. Numbered lines in a and b (right) indicate locations of molecular weight standards.

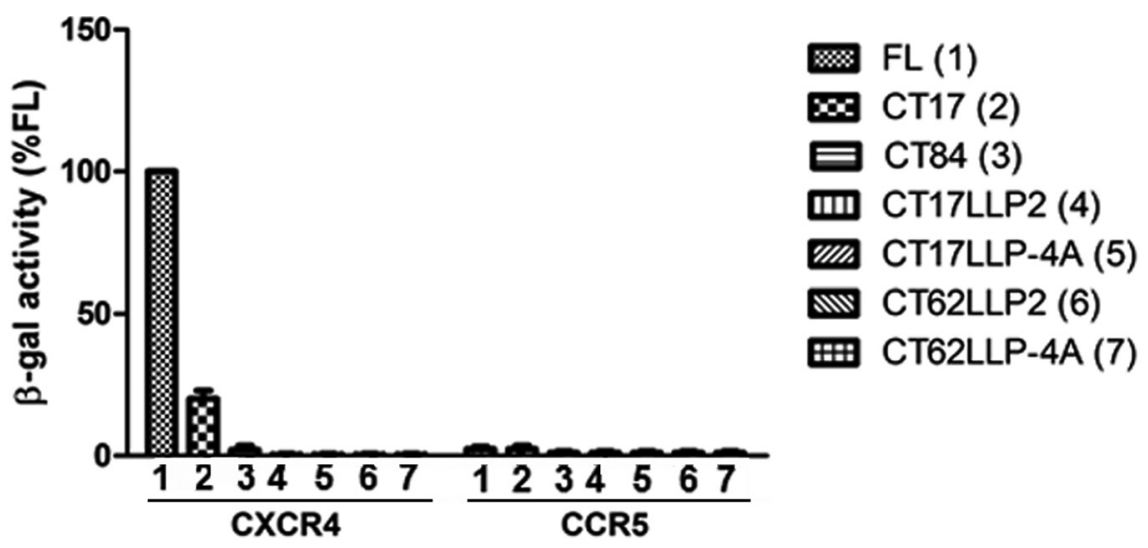

Fig. 3

Cell fusion activity of Env 92 UG046 with FL or truncated CT sequences

NIH3T3 cells were infected with a vaccinia vTF7-3 virus and then transfected by T7 plasmid constructs. 3T3T4R5 or 3T3T4X4 cells were infected with IHD-J vaccinia virus and transfected with a reporter gene construct. At $18 \mathrm{hr}$ post-transfection, the two populations were mixed and incubated, after which cell fusion was quantitated by a colorimetric lysate assay. The data shown are the percentages of the ß-Gal activity observed in WT (FL Env)-expressing cells. Data are plotted as the mean of three experiments. Error bars represent standard deviations.

did not affect levels of Env incorporation. All truncated Env proteins with the NL4-3 SP were found to be incorporated into pseudotyped virions at levels about 6 to $11 \%$ of the wild type FL Env (Fig. 4a,b). The mutant Env proteins (CT17LLP2,
CT17LLP2-4A, CT62LLP2, or CT62LLP2-4A) were found to be incorporated into pseudotyped virions at about the same levels as the parental CT17 or CT84 Env, but at a significantly lower level than FL Env. The intensity of the p24 protein 
(a)

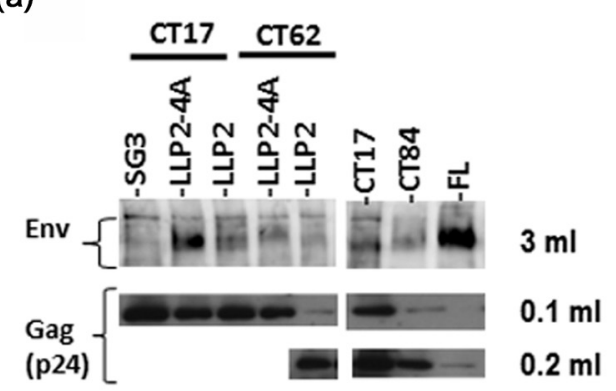

(b)

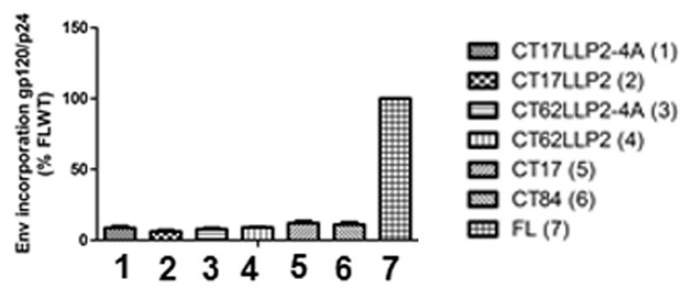

(c)

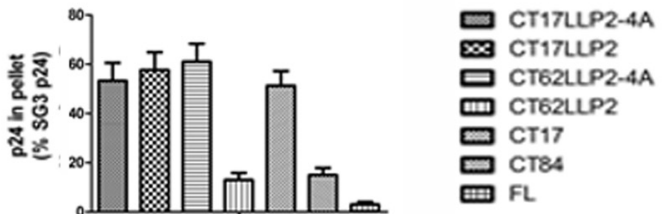

Fig. 4

Effects of CT domains on production and Env incorporation into pseudotyped virions

(a) Virions pseudotyped with 92UG046 Env proteins were obtained by co-transfection of 293T cells with Env plasmid constructs and with a plasmid expressing the env-minus molecular clone SG3 (HIV-1) using a ratio of Env plasmids to SG3 of $3: 1$ and collected and purified after 3 days. The same amount of medium $(0.1 \mathrm{ml}, 0.2 \mathrm{ml}, 3 \mathrm{ml})$ from equal amounts of cells was used in all samples except for SG3, which used 1/5 of the amount of medium of other samples. The samples were analyzed by $8 \%$ SDS-PAGE and Western blotting by using a polyclonal antibody from human plasma (HIV-Ig) for analysis of Gag and Env proteins. The amounts of proteins were quantitated by densitometer analysis (NIH Image version 1.54) for each mutant (b, c). The Env/Gag ratio was determined and indicated as \% of the FL Env level (100\%) (b). The Gag level was determined and indicated as \% of the SG3 Gag level $(100 \%)(c)$. Data are plotted as the mean of three experiments. Error bars represent standard deviations.

bands in CT17LLP2, CT17LLP2-4A, CT62LLP2-4A pseudotyped virions was about a 30 -fold higher than the p24 level in virus pseudotyped with FL Env (Fig. 4c). In contrast, the p24 levels of virions pseudotyped with CT84 or CT62LLP2 were about 5-fold higher than with FL Env (Fig. 4c). Taken together, the results indicate that $\mathrm{FL}$ and truncated Env were (a)

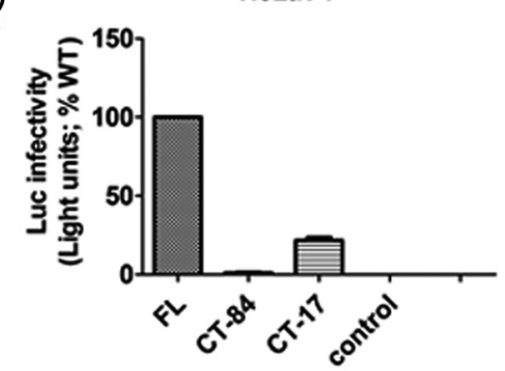

(c)

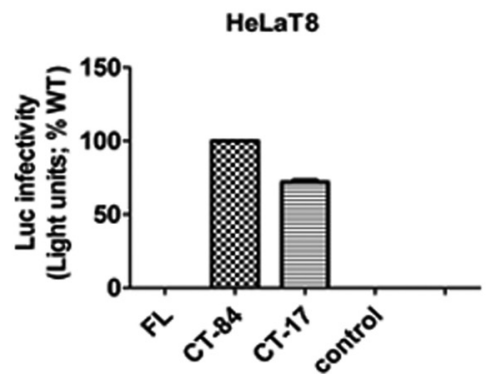

(b)

\section{TZM-bl cells (CD4-high)}

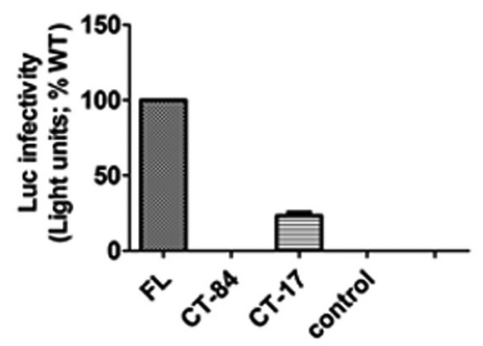

(d)

HeLa

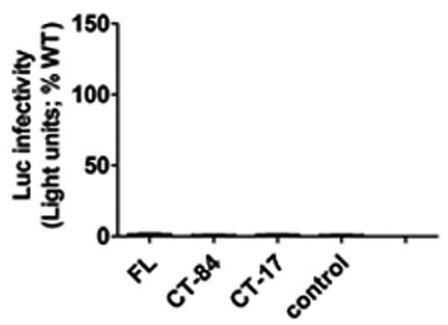

Fig. 5

Entry of virions pseudotyped with HIV-1 92UG046 Env with FL or truncated CT into TZM-bl, HeLaT4, and HeLaT8 cells Single-round infections were performed in HeLa or derivative HeLa cells with CD4 or CD8 receptors using a replication-defective NL4-3 virus bearing the luciferase reporter pseudotyped with FL CT84, CT17 envelopes. Luciferase activity was calculated after comparing luciferase activity from extracts of cells infected with FL $(100 \%)(a, b)$ or with CT84 $(100 \%)(c)$. Uninfected cells were used as control. The results are shown with the standard deviation $(\mathrm{n}=3)$. 
incorporated into pseudotyped virions at different levels and the LLP sequence selectively affected this process.

Effects of CT length on entry of virions pseudotyped with 92UG046 Env

To compare the entry of virions pseudotyped with FL or truncated Env 92UG046 into cells with different receptors, we compared HeLaT4 cells (expressing low CD4 levels), TZM-bl cells (expressing high CD4 levels) and HeLaT8 cells (expressing CD8). HIV infectivity was determined by $\beta$-galactosidase activity of a modified $\beta$-galactosidase gene driven by the HIV-1 long terminal repeat (LTR) promoter and transactivation by the viral Tat protein in TZM-bl cells (Kimpton and Emerman, 1992) or by infection with luciferase-encoding HIV-1 pseudotyped virions (He et al., 1995). Virions pseudotyped with CT17 92UG046 Envs were able to enter cells with CD4 or CD8 receptors (HeLaT4, HeLaT8 and TZM-bl cells) (Fig. 5). Virions pseudotyped with FL 92UG046 Env were able to enter cells with CD4 receptors (HeLaT4 or TZM-bl cells) and infected CD4 $4^{+}$-HeLa cells with high efficiency. In contrast, virions pseudotyped with CT84 Env were only able to infect HeLaT8 cells by using CD8-mediated entry. However, the efficiency of infection of the viruses pseudotyped with CT84 Env was relatively low, about 100 relative luciferase units per $1 \times 10^{4}$ cells. We also observed that replacement of the SP sequence by that of NL4-3 reduced the infectivity of Env FL pseudotyped virions about 1000-fold, but no such effect was observed with CT84 Env pseudotyped virions. The results indicate that the CT domain has a significant effect on productive entry of 92UG046 HIV-1 Env pseudotyped virions into HeLa cells with specific receptors, and that the length of the CT domain can confer an effect on infectivity.

Effects of antibodies on entry of FL or truncated Env pseudotyped virions in CD4-high, CD4-low, or CD8 HeLa cells

We hypothesized that the CT84 truncation of the cytoplasmic domain confers a conformational change to the external domain of Env, which is responsible for the observed differences in tropism of the 92UG046 Env proteins. To further investigate the effect of the CT84 truncation on receptor specificity of the Env protein, we used a blocking assay for entry into HeLaT4 or HeLaT8 cells by anti-CD4 (clones Q4120, RPA-T4) or anti-CD8 monoclonal antibodies. Nondividing cells were arrested in the $\mathrm{G}_{1}-\mathrm{S}$ phase of the cell cycle by using $2 \mu \mathrm{g} / \mathrm{ml}$ of aphidicolin (Huberman, 1981). In HeLaT8 cells treated with anti-CD8 antibodies, infection by virions pseudotyped with CT84 Env was reduced compared to untreated HeLaT8 cells (Fig. 6a,b). However, we observed that anti-CD8 antibody had no effect on infection using viri- ons pseudotyped with FL Env. Anti-CD4 (Q4120, RPA-T4) antibodies did not block entry of pseudotyped virions with modified or unmodified Envs in HeLaT4 or HeLaT8 cells. In contrast to RPA-T4, Q4120 Ab was able to block infection by virions pseudotyped with FL Env in TZM-bl cells (Fig. 6d). We also observed that anti-CD8 partially blocked the entry of CT62LLP2 pseudotyped virions into HeLaT8 cells but had no effect on the entry of virions pseudotyped with CT62LLP2-4A (Fig. 6c), which may reflect disruption of the helical sequence of the LLP2 domain of CT62LLP2 Env. The anti-CD4 and anti-CD8 antibodies were also ineffective at blocking infection by virions pseudotyped with Env CT17. These results further indicate that the structure and length of the CT domain can modify the receptor specificity for infection by HIV-1 Env.

\section{Effect of antibodies on binding of FL or truncated Env} pseudotyped virions to CD4 or CD8 receptors

We compared effects of antibody on binding of FL and truncated Env pseudotyped virions to CD4 or CD8 proteins to understand the mechanism by which the CT domain modulates the efficiency of infection of cells. The binding of pseudotyped virions was measured by competitive binding ELISA in the presence of anti-CD4 or anti-CD8 monoclonal antibodies or a control antibody. The CT84 and CT17 pseudotyped virions efficiently recognized CD4 or CD8 molecules, whereas binding of FL Env pseudotyped virions was blocked by anti-CD4 antibodies (Fig. 7a,b). The binding of truncated CT17, CT84 and FL Env pseudotyped virions to CD4 was reduced in the presence of anti-CD4 (Q4120) antibodies (about 35\% to 75\% reduction) but no such effect was seen with the RPA-T4 antibody (Fig. 7c,d,e). Notably, binding of CT84 Env pseudotyped virions to CD8 was reduced in the presence of anti-CD8 antibodies (about $74 \%$ reduction).

\section{Discussion}

Previous studies have shown that truncation of the CT can have various biological effects on the HIV Env protein, including increasing its expression levels on the cell surface, enhancing incorporation into VLPs, and promoting cell fusion activity (Johnston et al., 1993; Ritter et al., 1993; Spies and Compans, 1994; Vzorov and Compans, 1996; Zingler and Littman, 1993). The main goal of the present study was to investigate the possible effects of changes in the cytoplasmic domain on the alteration of HIV cell tropism. Our hypothesis that the cytoplasmic tail plays an important role in determining HIV-1 tropism is based in part on previous observations: (i) truncation of the CT domain alters the conformation of the external domain (Spies et al., 1994; Vzorov and Compans, 

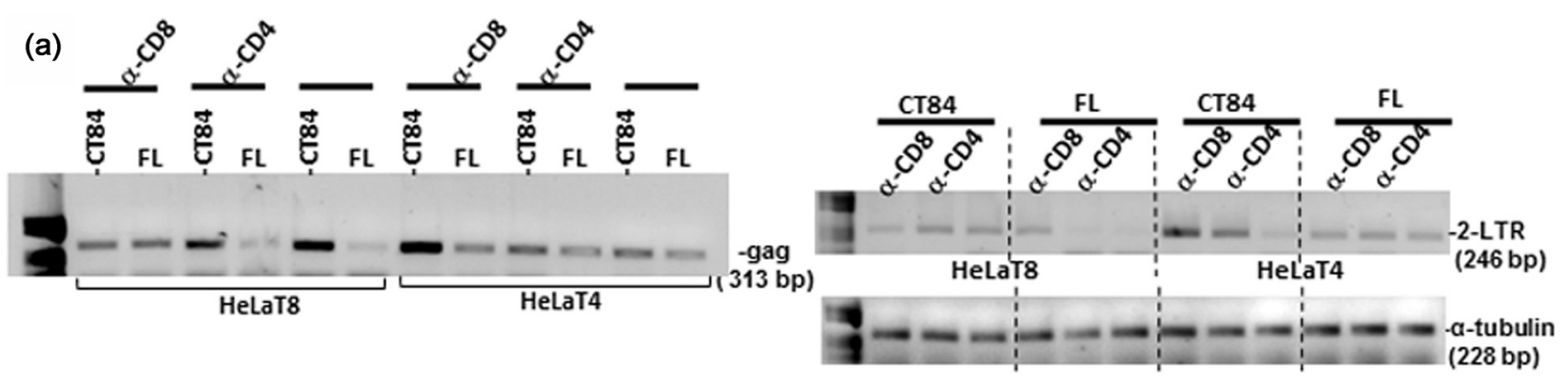

(b)

HeLaT4

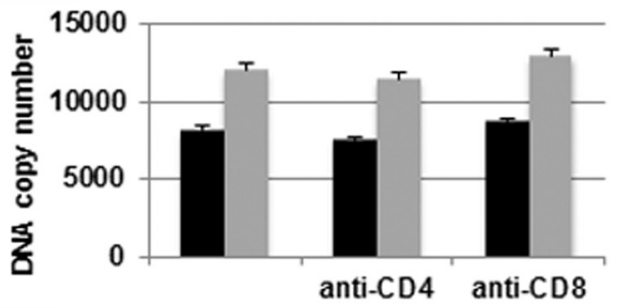

(c)

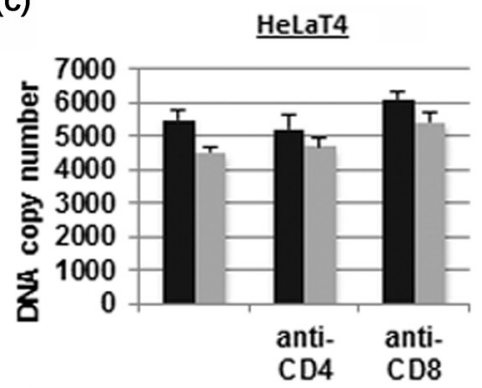

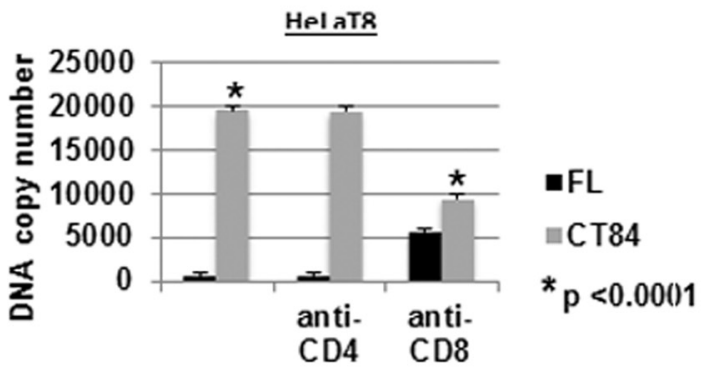

HeLaT8

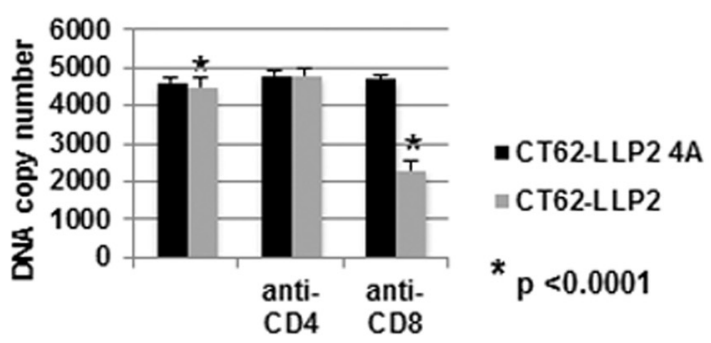

(d)

92UG046 FL Env

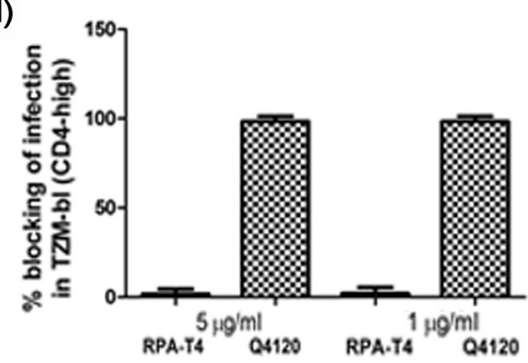

Fig. 6

Blocking of infection by 92 UG046 Env pseudotyped virions using mAbs RPA-T4, Q4120, or B9.11

Aphidicolin- treated HeLaT4 or HeLaT8 (a, b, c) or untreated TZM-bl (d) cells were incubated with antibodies for $1 \mathrm{~h}$ at $4^{\circ} \mathrm{C}$ and infected with CT84 or FL 92UG046 Env pseudotyped virions with 1 or 10 infectious index (IU/ng p24 Gag), respectively (a, b, c), or 30 infectious particles of pseudotyped virions per well (d). (a, left panel) viral entry was determined by PCR amplification for the HIV SG3 RGag region 24 hr post-infection. (a, right panel) Viral entry determined by PCR amplification for the HIV 2-LTR circle $24 \mathrm{hr}$ post-infection; PCR analysis of the $\alpha$-tubulin gene in cell lysates was used to standardize DNA recovery. (b, c) Viral entry determined by RT-PCR amplification for the HIV SG3 RGag region 24 hr post-infection. (d) Viral infectivity determined by a single-cycle (TZM-bl) infectivity assay. Data are plotted as the mean of three experiments and error bars represent standard deviations (b, c, d). Asterisks denote significant differences of the HIV-1 DNA copy numbers in HeLaT8 cells treated with anti-CD8 Abs compared to untreated HeLaT8 cells infected by CT84 or CT162LLP2 ( $<0.0001$ by unpaired Student's $t$-test). 
(a)

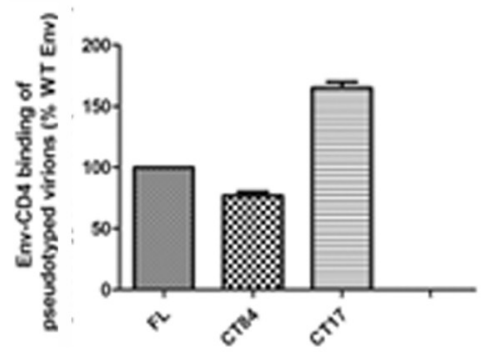

(c)

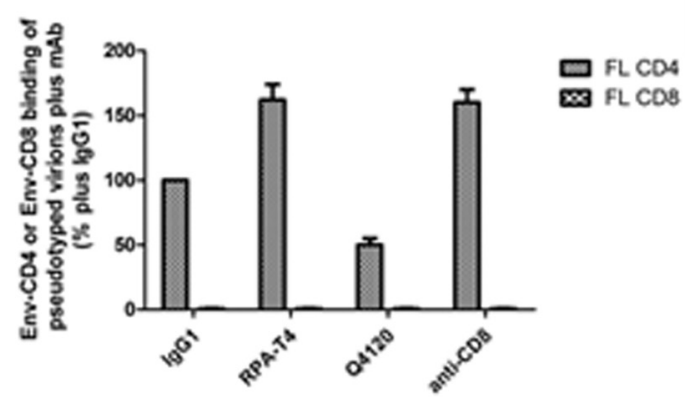

(b)

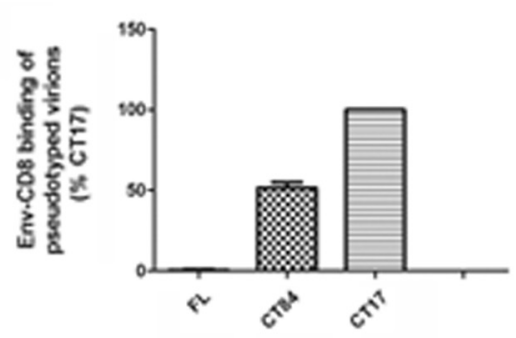

(d)

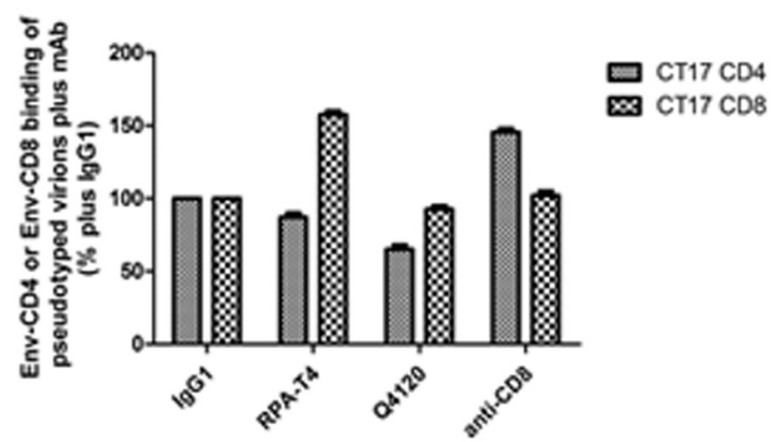

(e)

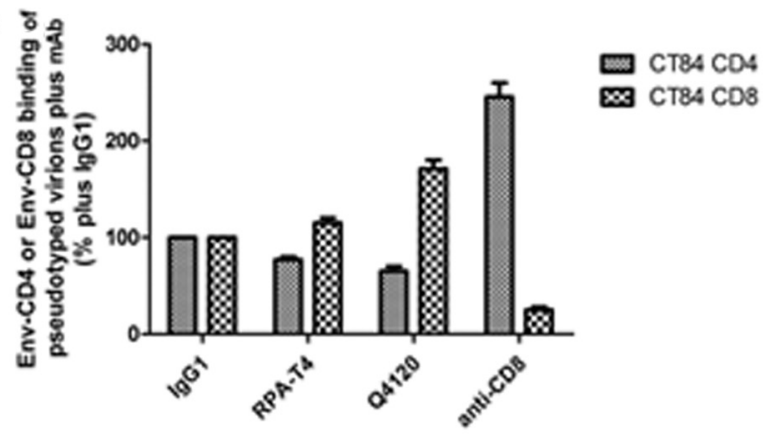

Fig. 7

Effects of antibodies on binding of FL or truncated Env pseudotyped virions to CD4 or CD8

A 96-well plate was coated with OKT4 (a, c, d, e) or OKT8 (b, c, d, e) antibody and with soluble CD4 or CD8, and incubated with virions pseudotyped with Env in the presence of anti-CD4 or CD8 antibodies. After extensive washes the bound virions were detected by biotin-conjugated polyclonal antibody to HIV-1. Results represent percentage of bound virions in the presence of anti-CD4 or anti-CD8 Ab compared to that observed for bound virions in the presence of mouse IgG1 (100\%). The results are shown with the standard deviation $(\mathrm{n}=3)$.

2000); (ii) changes in phenotypic characteristics of the SU are affected by CT length (Vzorov et al., 2005); (iii) the length and sequence of the CT domain can impact viral biological properties including trimer stability, syncytia formation activity and infectivity (Bonavia et al., 2005; Edwards et al., 2001; Taylor et al., 2008; Vzorov and Compans, 2011; Wyss et al., 2005). In this study we show that the tropism of FL 92UG046 Env changes from a CD4-high phenotype to a CD8-tropic phenotype when a truncated form of Env is used to initiate an infection. The precise sequence, which were important to change the 92UG046 Env tropism were not determined in previous studies (Saha et al., 2005;
Zerhouni et al., 2004). Moreover, the authors reported that CD4-independent virus isolates (including HIV-1 92UG046$\mathrm{T} 8$ ), which they obtained after infection of purified $\mathrm{CD}^{+} \mathrm{T}$ cells, were not inhibited by anti-CD8 antibodies, in contrast to previously reported CD ${ }^{+} \mathrm{T}$ cell tropic viruses (Saha et al., 2001). In one study CD4-independent variants were isolated from 7 of 12 patients through infection of purified $\mathrm{CD}^{+} \mathrm{T}$ cells, and one CD4-independent variant isolated from a patient (92UG046-T8) had a sharply truncated cytoplasmic tail (CT84) due to a point mutation (Zerhouni et al., 2004). To investigate the sequences responsible for differences in tropism, we compared two types of CT truncations: an exten- 
sive truncation, which deleted membrane-interactive regions such as amphipathic helical regions LLP-1, LLP-2, and LLP-3 (Chen et al., 2001; Chernomordik et al., 1994; Comardelle et al., 1997; Kalia et al., 2003; Miller et al., 1993; Srinivas et al., 1992; Venable et al., 1989) and shorter truncations located within specific amphipathic helical regions (60 to 150 aa). Many isolates of HIV-2 and SIV contain stop codons that truncate the CT to within 15 to 20 aa of the membranespanning domain, which have been shown to occur upon passage of the virus in culture (Hirsch et al., 1989; Kodama et al., 1989; Mulligan et al., 1992). Macaques inoculated by SIV1A11 with a cytoplasmic tail of 18 aa demonstrated transient viremia (Luciw et al., 1992), while infection of macaques by an SIV17E variant with a longer cytoplasmic tail of 48 aa led to disseminated infection (Mankowski et al., 1997). In HIV-1, some variation of size in the Env CT has also been observed. HIV-1 (KB-1) carrying an 18aa Env CT was isolated from a Japanese male hemophiliac (Shimizu et al., 1992). HIV-1 isolates that show CD4 independence bear extensive truncations in their gp41 CTs, located prior to the membrane-interactive regions (Edwards et al., 2001, 2002). SIV with a truncated Env-CT17 exhibits a defect in early steps of replication in non-dividing cells, and demonstrates an extended range of cells susceptible to infection (Vzorov et al., 2007).

$\mathrm{CD}^{+}$or $\mathrm{CD}^{+} \mathrm{HeLa}$ cell clones provide a sensitive system for quantitatively comparing infectivity of M-tropic and T-cell-tropic isolates of HIV-1, including primary patient isolates (Platt et al., 1998). HIV binds at higher levels to HeLa cells than to other cells. A low gp120-CD4 interaction is masked by a more avid gp120-heparan sulfate interaction in HeLaT4 cells (Mondor et al., 1998). Viral entry studies have also been conducted using HeLa cell lines expressing different levels of CD4 and CCR5 viral receptors (Platt et al., 1998). Reduced CD4 levels were a significant barrier for entry of cells by HIV-1 clones with certain V1/V2 modifications (Walter et al., 2005).

We observed differences in entry levels of FL, CT84, CT17, or modified Env pseudotyped virions in HeLaT4, HeLaT8 or TZM-bl cells. We found that the addition of the LLP-2 sequence to the truncated CT62 construct resulted in the ability to infect HeLaT8 cells, while the corresponding virions pseudotyped with this construct containing $2 \mathrm{~L}$ and 2 I to $4 \mathrm{~A}$ mutations were unable to infect these cells, indicating that the helical structure of LLP2 sequence is important for this process. Moreover, the LLP sequence and length of the CT domain may affect selective incorporation of Env glycoproteins into viral particles. Most importantly, we observed that LLP sequences in the CT domain may change the receptor specificity of the Env protein. These results support the conclusion that the LLP-2 sequence and the structure of the cytoplasmic tail play important roles in determining the receptor-specificity of the 92UG046 HIV-1
Env protein. Anti-CD8 antibodies were only able to inhibit entry of CT84 pseudotyped virions in HeLaT8 cells, suggesting that the CT84 Env determines the receptor specificity for infection of these cells. Anti-CD8 antibodies did not inhibit HIV-1 92UG046-T8 entry in CD8 ${ }^{+}$cells in a previous study (Zerhouni et al., 2004) but a blocking effect was demonstrated in the present study. This difference may be due to using virions pseudotyped with a cloned CT84 Env in our experiments. The incomplete blockage observed with antiCD8 antibodies may also be due to ability of HIV-1 virions to bypass receptor requirements and enter through endocytic pathways (Fredericksen et al., 2002; Wei et al., 2005).

In conclusion, we believe that the present results provide the first evidence that a specific sequence in the CT domain can modulate viral receptor specificity of HIV-1 Env. We hypothesize that the CT84 truncation in the CT domain confers CD8-tropism by inducing conformational changes in functional regions of gp120. Such changes may enhance exposure of receptor-binding segments in gp120, resulting in viruses that differ in their cell tropism and biological properties.

Acknowledgements. We thank Dahnide Taylor for her technical assistance and Erin-Joi Collins for her assistance with the preparation and submission of this manuscript. AV would like to thank Gregory Melikian for helpful discussion. This study was supported by a grant from the National Institutes of Health (AI090480) and Emory CFAR grant (P30-AI-50409). The reagents were obtained through the NIH ARRRP. The funders had no role in study design, data collection and analysis, decision to publish, or preparation of the manuscript.

\section{References}

Berlioz-Torrent C, Shacklett BL, Erdtmann L, Delamarre L, Bouchaert I, Sonigo P, Dokhelar MC, Benarous R (1999): Interactions of the cytoplasmic domains of human and simian retroviral transmembrane proteins with components of the clathrin adaptor complexes modulate intracellular and cell surface expression of envelope glycoproteins. J. Virol. 73, 1350-1361.

Bonavia A, Bullock BT, Gisselman KM, Margulies BJ, Clements JE (2005): A single amino acid change and truncated $\mathrm{TM}$ are sufficient for simian immunodeficiency virus to enter cells using CCR5 in a CD4-independent pathway. Virology 341, 12-23. http://dx.doi.org/10.1016/j. virol.2005.07.001

Bowers K, Pelchen-Matthews A, Honing S, Vance PJ, Creary L, Haggarty BS, Romano J, Ballensiefen W, Hoxie JA, Marsh M (2000): The simian immunodeficiency virus envelope glycoprotein contains multiple signals that regulate its cell surface expression and endocytosis. Traffic 1, 661-674. http://dx.doi.org/10.1034/j.1600-0854.2000.010810.x

Chen SS, Lee SF, Wang CT (2001): Cellular membrane-binding ability of the C-terminal cytoplasmic domain of human immunodeficiency virus type 1 envelope transmembrane 
protein gp41. J. Virol. 75, 9925-9938. http://dx.doi. org/10.1128/JVI.75.20.9925-9938.2001

Chernomordik L, Chanturiya AN, Suss-Toby E, Nora E, Zimmerberg J (1994): An amphipathic peptide from the C-terminal region of the human immunodeficiency virus envelope glycoprotein causes pore formation in membranes. J. Virol. 68, 7115-7123.

Comardelle AM, Norris CH, Plymale DR, Gatti PJ, Choi B, Fermin CD, Haislip AM, Tencza SB, Mietzner TA, Montelaro RC, Garry RF (1997): A synthetic peptide corresponding to the carboxy terminus of human immunodeficiency virus type 1 transmembrane glycoprotein induces alterations in the ionic permeability of Xenopus laevis oocytes. AIDS Res. Hum. Retroviruses 13, 1525-1532. http://dx.doi. org/10.1089/aid.1997.13.1525

Desire N, Dehee A, Schneider V, Jacomet C, Goujon C, Girard PM, Rozenbaum W, Nicolas JC (2001): Quantification of human immunodeficiency virus type 1 proviral load by a TaqMan real-time PCR assay. J. Clin. Microbiol. 39, 1303-1310. http:// dx.doi.org/10.1128/JCM.39.4.1303-1310.2001

Edwards TG, Hoffman TL, Baribaud F, Wyss S, LaBranche CC, Romano J, Adkinson J, Sharron M, Hoxie JA, Doms RW (2001): Relationships between CD4 independence, neutralization sensitivity, and exposure of a CD4-induced epitope in a human immunodeficiency virus type 1 envelope protein. J. Virol. 75, 5230-5239. http://dx.doi. org/10.1128/JVI.75.11.5230-5239.2001

Edwards TG, Wyss S, Reeves JD, Zolla-Pazner S, Hoxie JA, Doms RW, Baribaud F (2002): Truncation of the cytoplasmic domain induces exposure of conserved regions in the ectodomain of human immunodeficiency virus type 1 envelope protein. J. Virol. 76, 2683-2691. http://dx.doi. org/10.1128/JVI.76.6.2683-2691.2002

Fredericksen BL, Wei BL, Yao J, Luo T, Garcia JV (2002): Inhibition of endosomal/lysosomal degradation increases the infectivity of human immunodeficiency virus. J. Virol. 76, 11440-11446. http://dx.doi.org/10.1128/JVI.76.22.1144011446.2002

He J, Choe S, Walker R, Di Marzio P, Morgan DO, Landau NR (1995): Human immunodeficiency virus type 1 viral protein $\mathrm{R}$ (Vpr) arrests cells in the G2 phase of the cell cycle by inhibiting p34cdc2 activity. J. Virol. 69, 6705-6711.

Hirsch VM, Edmondson P, Murphey-Corb M, Arbeille B, Johnson PR, Mullins JI (1989): SIV adaptation to human cells. Nature 341, 573-574. http://dx.doi. org/10.1038/341573a0

Huberman JA (1981): New views of the biochemistry of eucaryotic DNA replication revealed by aphidicolin, an unusual inhibitor of DNA polymerase alpha. Cell 23, 647-648. http://dx.doi.org/10.1016/0092-8674(81)90426-8

Johnston PB, Dubay JW, Hunter E (1993): Truncations of the simian immunodeficiency virus transmembrane protein confer expanded virus host range by removing a block to virus entry into cells. J. Virol. 67, 3077-3086.

Kalia V, Sarkar S, Gupta P, Montelaro RC (2003): Rational sitedirected mutations of the LLP-1 and LLP-2 lentivirus lytic peptide domains in the intracytoplasmic tail of human immunodeficiency virus type $1 \mathrm{gp} 41$ indicate common functions in cell-cell fusion but distinct roles in virion envelope incorporation. J. Virol. 77, 3634-3646. http:// dx.doi.org/10.1128/JVI.77.6.3634-3646.2003

Kim SS, You XJ, Harmon ME, Overbaugh J, Fan H (2001): Use of helper-free replication-defective simian immunodeficiency virus-based vectors to study macrophage and T tropism: evidence for distinct levels of restriction in primary macrophages and a T-cell line. J. Virol. 75, 2288-2300. http:// dx.doi.org/10.1128/JVI.75.5.2288-2300.2001

Kimpton J, Emerman M (1992): Detection of replication-competent and pseudotyped human immunodeficiency virus with a sensitive cell line on the basis of activation of an integrated beta-galactosidase gene. J. Virol. 66, 2232-2239.

Kodama T, Wooley DP, Naidu YM, Kestler HW, 3rd, Daniel MD, Li Y, Desrosiers RC (1989): Significance of premature stop codons in env of simian immunodeficiency virus. J. Virol. 63, 4709-4714.

Luciw PA, Shaw KE, Unger RE, Planelles V, Stout MW, Lackner JE, Pratt-Lowe E, Leung NJ, Banapour B, Marthas ML (1992): Genetic and biological comparisons of pathogenic and nonpathogenic molecular clones of simian immunodeficiency virus (SIVmac). AIDS Res. Hum. Retroviruses. 8, 395-402. http://dx.doi.org/10.1089/ aid.1992.8.395

Mankowski JL, Flaherty MT, Spelman JP, Hauer DA, Didier PJ, Amedee AM, Murphey-Corb M, Kirstein LM, Munoz A, Clements JE, Zink MC (1997): Pathogenesis of simian immunodeficiency virus encephalitis: viral determinants of neurovirulence. J. Virol. 71, 6055-6060.

Miller MA, Cloyd MW, Liebmann J, Rinaldo CR, Jr., Islam KR, Wang SZ, Mietzner TA, Montelaro RC (1993): Alterations in cell membrane permeability by the lentivirus lytic peptide (LLP-1) of HIV-1 transmembrane protein. Virology 196, 89-100. http://dx.doi.org/10.1006/viro.1993.1457

Mondor I, Ugolini S, Sattentau QJ (1998): Human immunodeficiency virus type 1 attachment to HeLa CD4 cells is CD4 independent and gp120 dependent and requires cell surface heparans. J. Virol. 72, 3623-3634.

Mulligan MJ, Yamshchikov GV, Ritter GD, Jr., Gao F, Jin MJ, Nail CD, Spies CP, Hahn BH, Compans RW (1992): Cytoplasmic domain truncation enhances fusion activity by the exterior glycoprotein complex of human immunodeficiency virus type 2 in selected cell types. J. Virol. 66, 3971-3975.

Nussbaum O, Broder CC, Berger EA (1994): Fusogenic mechanisms of enveloped-virus glycoproteins analyzed by a novel recombinant vaccinia virus-based assay quantitating cell fusion-dependent reporter gene activation. J. Virol. 68, 5411-5422.

Platt EJ, Wehrly K, Kuhmann SE, Chesebro B, Kabat D (1998): Effects of CCR5 and CD4 cell surface concentrations on infections by macrophagetropic isolates of human immunodeficiency virus type 1. J. Virol. 72, 2855-2864.

Ritter GD, Jr., Mulligan MJ, Lydy SL, Compans RW (1993): Cell fusion activity of the simian immunodeficiency virus envelope protein is modulated by the intracytoplasmic domain. Virology 197, 255-264. http://dx.doi.org/10.1006/ viro.1993.1586 
Saha K, Yan H, Nelson JA, Zerhouni-Layachi B (2005): Infection of human and non-human cells by a highly fusogenic primary CD4-independent HIV-1 isolate with a truncated envelope cytoplasmic tail. Virology 337, 30-44. http:// dx.doi.org/10.1016/j.virol.2005.04.003

Saha K, Zhang J, Gupta A, Dave R, Yimen M, Zerhouni B (2001): Isolation of primary HIV-1 that target CD8+ T lymphocytes using CD8 as a receptor. Nat. Med. 7, 65-72. http://dx.doi.org/10.1038/83365

Sauter MM, Pelchen-Matthews A, Bron R, Marsh M, LaBranche CC, Vance PJ, Romano J, Haggarty BS, Hart TK, Lee WM, Hoxie JA (1996): An internalization signal in the simian immunodeficiency virus transmembrane protein cytoplasmic domain modulates expression of envelope glycoproteins on the cell surface. J. Cell Biol. 132, 795-811. http://dx.doi.org/10.1083/jcb.132.5.795

Schmidtmayerova H, Alfano M, Nuovo G, Bukrinsky M (1998): Human immunodeficiency virus type 1 T-lymphotropic strains enter macrophages via a CD4- and CXCR4mediated pathway: replication is restricted at a postentry level. J. Virol. 72, 4633-4642.

Shimizu H, Hasebe F, Tsuchie H, Morikawa S, Ushijima H, Kitamura T (1992): Analysis of a human immunodeficiency virus type 1 isolate carrying a truncated transmembrane glycoprotein. Virology 189, 534-546. http://dx.doi. org/10.1016/0042-6822(92)90577-C

Spies CP, Compans RW (1994): Effects of cytoplasmic domain length on cell surface expression and syncytium-forming capacity of the simian immunodeficiency virus envelope glycoprotein. Virology 203, 8-19. http://dx.doi. org/10.1006/viro.1994.1449

Spies CP, Ritter GD, Jr., Mulligan MJ, Compans RW (1994): Truncation of the cytoplasmic domain of the simian immunodeficiency virus envelope glycoprotein alters the conformation of the external domain. J. Virol. 68, 585-591.

Srinivas SK, Srinivas RV, Anantharamaiah GM, Segrest JP, Compans RW (1992): Membrane interactions of synthetic peptides corresponding to amphipathic helical segments of the human immunodeficiency virus type-1 envelope glycoprotein. J. Biol. Chem. 267, 7121-7127.

Taylor BM, Foulke JS, Flinko R, Heredia A, DeVico A, Reitz M (2008): An alteration of human immunodeficiency virus gp41 leads to reduced CCR5 dependence and CD4 independence. J. Virol. 82, 5460-5471. http://dx.doi. org/10.1128/JVI.01049-07

Tilton JC, Doms RW (2010): Entry inhibitors in the treatment of HIV-1 infection. Antiviral Res. 85, 91-100. http://dx.doi. org/10.1016/j.antiviral.2009.07.022

Venable RM, Pastor RW, Brooks BR, Carson FW (1989): Theoretically determined three-dimensional structures for amphipathic segments of the HIV-1 gp41 envelope protein. AIDS Res. Hum. Retroviruses. 5 7-22. http://dx.doi. org/10.1089/aid.1989.5.7

Vzorov AN, Compans RW (1996): Assembly and release of SIV env proteins with full-length or truncated cytoplasmic domains. Virology 221, 22-33. http://dx.doi.org/10.1006/ viro.1996.0349
Vzorov AN, Compans RW (2000): Effect of the cytoplasmic domain of the simian immunodeficiency virus envelope protein on incorporation of heterologous envelope proteins and sensitivity to neutralization. J. Virol. 74, 8219-8225. http://dx.doi.org/10.1128/JVI.74.18.8219$\underline{8225.2000}$

Vzorov AN, Compans RW (2011): Effects of stabilization of the gp41 cytoplasmic domain on fusion activity and infectivity of SIVmac239. AIDS Res. Hum. Retroviruses 27, 1213-1222. http://dx.doi.org/10.1089/aid.2010.0321

Vzorov AN, Gernert KM, Compans RW (2005): Multiple domains of the SIV Env protein determine virus replication efficiency and neutralization sensitivity. Virology 332, 89-101. http://dx.doi.org/10.1016/j.virol.2004.10.044

Vzorov AN, Weidmann A, Kozyr NL, Khaoustov V, Yoffe B, Compans RW (2007): Role of the long cytoplasmic domain of the SIV Env glycoprotein in early and late stages of infection. Retrovirology 4, 94. http://dx.doi.org/10.1186/17424690-4-94

Waheed AA, Ablan SD, Roser JD, Sowder RC, Schaffner CP, Chertova E, Freed EO (2007): HIV-1 escape from the entryinhibiting effects of a cholesterol-binding compound via cleavage of gp41 by the viral protease. Proc. Natl. Acad. Sci. USA 104, 8467-8471. http://dx.doi.org/10.1073/ pnas. 0701443104

Walter BL, Wehrly K, Swanstrom R, Platt E, Kabat D, Chesebro B (2005): Role of low CD4 levels in the influence of human immunodeficiency virus type 1 envelope $\mathrm{V} 1$ and V2 regions on entry and spread in macrophages. J. Virol. 79, 4828-4837. http://dx.doi.org/10.1128/JVI.79.8.4828$\underline{4837.2005}$

Wei BL, Denton PW, O'Neill E, Luo T, Foster JL, Garcia JV (2005): Inhibition of lysosome and proteasome function enhances human immunodeficiency virus type 1 infection. J. Virol. 79, 5705-5712. http://dx.doi.org/10.1128/JVI.79.9.5705$\underline{5712.2005}$

Wei X, Decker JM, Liu H, Zhang Z, Arani RB, Kilby JM, Saag MS, Wu X, Shaw GM, Kappes JC (2002): Emergence of resistant human immunodeficiency virus type 1 in patients receiving fusion inhibitor (T-20) monotherapy. Antimicrob. Agents Chemother. 46, 1896-1905. http:// dx.doi.org/10.1128/AAC.46.6.1896-1905.2002

Wyss S, Dimitrov AS, Baribaud F, Edwards TG, Blumenthal R, Hoxie JA (2005): Regulation of human immunodeficiency virus type 1 envelope glycoprotein fusion by a membrane-interactive domain in the gp 41 cytoplasmic tail. J. Virol. 79, 12231-12241. http://dx.doi.org/10.1128/ JVI.79.19.12231-12241.2005

Zerhouni B, Nelson JA, Saha K (2004): Isolation of CD4-independent primary human immunodeficiency virus type 1 isolates that are syncytium inducing and acutely cytopathic for CD8+ lymphocytes. J. Virol. 78, 1243-1255. http:// dx.doi.org/10.1128/JVI.78.3.1243-1255.2004

Zingler K, Littman DR (1993): Truncation of the cytoplasmic domain of the simian immunodeficiency virus envelope glycoprotein increases env incorporation into particles and fusogenicity and infectivity. J. Virol. 67, 2824-2831. 